\title{
Shape-from-Shading for Highlighted Surfaces
}

\author{
Hossein Ragheb * and Edwin R. Hancock \\ Department of Computer Science, University of York \\ York YO10 5DD, UK \\ $\{$ hossein, erh\}@minster.cs. york. ac.uk
}

\begin{abstract}
One of the problems that hinders the application of conventional methods for shape-from-shading to the analysis of shiny objects is the presence of local highlights. The first of these are specularities which appear at locations on the viewed object where the local surface normal is the bisector of the light source and viewing directions. Highlights also occur at the occluding limb of the object where roughness results in backscattering from microfacets which protrude above the surface. In this paper, we consider how to subtract both types of highlight from shiny surfaces in order to improve the quality of surface normal information recoverable using shape-from-shading.
\end{abstract}

\section{Introduction}

Shape-from-shading (SFS) is concerned with recovering surface orientation from local variations in measured brightness [5]. The observation underpinning this paper is that although considerable effort has gone into the recovery of accurate surface geometry, existing SFS methods are confined to situations in which the reflectance is predominantly Lambertian. When the surface under study is shiny, then the estimated geometry may be subject to error. The main problem that can occur is that surface intensity highlights may lead to misestimation of surface curvature. The most familiar example here is that of surface specularities. These occur at locations on the surface where the local surface normal direction is the bisector of the light source and viewing directions For this reason, if specular highlights can be accurately located, then they can provide important cues that can be used to constrain the recovery of surface shape. However, there is a less well known effect that results in limb brightening. This is due to surface roughness and results from oblique scattering from microfacets that protrude above the limb perpendicular to the line of sight.

The problem of non-Labmertian and specular reflectance has been widely studied $[6,13]$. For instance, Healey and Binford [4] have shown how to simplify the Beckmann distribution [1] using a Gaussian approximation to the distribution of specular angle. This simplification can be used in conjunction with the Torrance and Sparrow model [14] to model intensity variations in the analysis of surface curvature. Several authors have looked critically at the physics underlying specular reflectance. For instance, Nayar, Ikeuchi and Kanade [9] have

\footnotetext{
* Sponsored by the university of Bu-Ali Sina, Hamedan, Iran.
} 
shown that the Torrance-Sparrow model [14] is applicable to the modelling of the specular lobe rather than the specular spike. Wolff [15] also has a model which combines diffuse and specular reflectance components, in which the parameters are chosen on the basis of the known physical properties of particular surfaces. In a series of recent papers, Lin and Lee have shown how specular reflections due to multiple light-sources can be located in multi-band imagery [7]. Finally, Nayar, Fang and Boult [10] have used polarisation filters to detect specular reflection.

There has also been a considerable body of work devoted to reflectance from rough surfaces. As noted above, this process is responsible for limb brightening. Oren and Nayar [11] have developed a model which can be used to account for reflectance from surfaces with a rough microfacet structure. Dana, Nayar, Van Ginneken and Koenderink [3] have catalogued the BRDF's for 3D surface textures. Recently, Magda, Kriegman, Zickler and Belhumeur [8] have commented on how shape can be recovered from surfaces with arbitrary BRDF's. Finally, Wolff [15] has shown how the Fresnel term can be used to model reflectance from a variety of surfaces.

In this paper our aim is to incorporate both specular and rough limb reflectance into the SFS process. This is a two-step process. First, we make estimates of the local surface normals using geometric constraints on the directions of Lambertian and specular reflectance to recover surface normal directions. The approach is a probabilistic one, which uses a mixture model to estimate the posterior mean direction of Lambertian and specular reflectance. Once the posterior mean surface normals are to hand, then we can perform photometric correction on the original image. This is again a two-step process. First, we subtract specularities using the Torrance-Sparrow (T-S) model. Second, we correct the residual non-specular component using the Oren-Nayar model. The result is a corrected Lambertian image from which both local specularities and limb-brightening effects are removed. By applying a Lamertian SFS algorithm to the corrected image, we obtain an improved estimate of the surface normal directions.

\section{Reflectance Geometry}

In this section we outline the geometry of the reflectance processes which underpin our SFS model. We adopt a two-component model in which the predominantly Lambertian surface reflectance exhibits local specular highlights.

In the case of Lambertian reflectance from a matte surface of constant albedo illuminated with a single collimated light-source, the observed intensity is independent of the viewing direction. Suppose that $\boldsymbol{L}$ is the unit-vector in the direction of the light source and that $\boldsymbol{N}_{L}(i, j)$ is the unit-vector in the surface normal at the pixel $(i, j)$. According to Lambert's law, the observed image intensity at the pixel with coordinates $(i, j)$ is $E(i, j)=\boldsymbol{N}_{L}(i, j) \cdot \boldsymbol{L}$

The second component of our reflectance process is concerned with modelling local specular highlights on the observed surface. For specular reflection the surface normal, the light source and the viewing directions are coplanar. The incidence angle is equal to the angle of specular reflectance (Figure 1a). Hence, 
the direction of the surface normal $\boldsymbol{N}_{S}^{(n)}$ is the bisector of the light source $(\boldsymbol{L})$ and the viewing $(\boldsymbol{V})$ directions and the unit-vector is $\quad \boldsymbol{N}_{S}^{(n)}=\frac{(\boldsymbol{L}+\boldsymbol{V})}{\|\boldsymbol{L}+\boldsymbol{V}\|}$.

\section{Bayesian Framework}

The aim in this paper is to develop a Bayes-decision scheme for separating the two reflectance modes [12]. In other words, we wish to compute the a posteriori probabilities of specular or Lambertian reflectance. The aim is to label pixels according to the reflectance mode from which they originated. The class identity for the pixel $(i, j)$ at iteration $n$ is denoted by $\omega_{i, j}^{(n)}$. The class-identity may be drawn from the set $\Omega=\{S, L\}$ where $S$ is the specular reflectance label and $L$ is the Lambertian reflectance label. For each image location, we maintain a specular surface normal and a Lambertian surface normal which satisfy the geometric constraints outlined in Section 2. At iteration $n$ of the algorithm the currently available estimates of the two surface normals are respectively $\boldsymbol{N}_{L}^{(n)}(i, j)$ and $\mathbf{N}_{S}^{(n)}(i, j)$. In the case of the specular component, the normal direction is in the direction of local specular reflection, and does not change with iteration number. In the case of Lambertian reflectance, the normal direction varies with iteration number, but is always projected to be positioned on the irradiance cone.

To develop our decision process, we require two probabilistic modelling ingredients. The first of these are separate probability density functions which can be used to represent the distributions of surface normals for the two reflectance components. We evaluate these densities at the posterior mean (PM) surface normal $\boldsymbol{M}^{(n)}(i, j)$ computed at iteration $n$. The reason for doing this is that the current values of the two normals are guaranteed to satisfy the geometric constraints outlined in Section 2. As a result, they will be associated with vanishing angular error. Accordingly, we let $q_{i, j}^{(n)}(L)=p\left(\boldsymbol{M}^{(n)}(i, j) \mid \omega_{i, j}^{(n)}=L\right)$ be the probability distribution for the PM surface normal under the Lambertian reflectance model. Similarly, we let $q_{i, j}^{(n)}(S)=p\left(\boldsymbol{M}^{(n)}(i, j) \mid \omega_{i, j}^{(n)}=S\right.$ ) denote the distribution function for the PM surface normal for the specular reflectance component.

The second probabilistic ingredient is a smoothness prior for the selected surface normal. This component of the model incorporates contextual information. Indexing the surface normals according to their pixel locations, suppose that $\Gamma_{i, j}^{(n)}=\left\{\boldsymbol{M}^{(n)}(k, l) \mid(k, l) \in G_{i, j}\right\}$ is the set of PM surface normals in the neighbourhood $G_{i, j}$ of the pixel $(i, j)$. We let $P_{i, j}^{(n)}(L)=P\left(\boldsymbol{N}_{L}^{(n)}(i, j) \mid \Gamma_{i, j}^{(n)}\right)$ be the conditional probability (or smoothness prior) of the Lambertian surface normal at the location $(i, j)$ given the field of surrounding PM surface normals. With these ingredients, then according to the iterated conditional modes, the probability that the pixel $(i, j)$ belongs to the Lambertian class at iteration $n$ is

$$
P\left(\omega_{i, j}^{(n)}=L \mid \boldsymbol{M}^{(n)}(i, j)\right)=\frac{q_{i, j}^{(n)}(L) P_{i, j}^{(n)}(L)}{\sum_{\Lambda \in \Omega} q_{i, j}^{(n)}(\Lambda) P_{i, j}^{(n)}(\Lambda)}
$$


The probability that the surface normal belongs to the specular class is the complement, These probabilities can be used to separate the two reflectance modes. With these probabilities to hand, we can update the estimate of the PM surface normal in the following manner

$$
\begin{aligned}
\boldsymbol{M}^{(n+1)}(i, j) & =\boldsymbol{N}_{S}^{(n)}(i, j) P\left(\omega_{i, j}^{(n)}=S \mid \boldsymbol{M}^{(n)}(i, j)\right) \\
& +\boldsymbol{N}_{L}^{(n)}(i, j) P\left(\omega_{i, j}^{(n)}=L \mid \boldsymbol{M}^{(n)}(i, j)\right)
\end{aligned}
$$

\section{Surface Normal Distribution}

To apply the MAP estimation scheme outlined in the previous section, we require probability distributions for the two surface normals together with the smoothness prior. For the specular surface normals, we use the Beckmann distribution to model the angle $\alpha=\cos ^{-1}\left(\boldsymbol{M}^{(n)}(i, j) \cdot \boldsymbol{N}_{S}^{(n)}(i, j)\right)$, between the PM surface normal $\boldsymbol{M}^{(n)}(i, j)$ and the predicted direction of the specular spike $\boldsymbol{N}_{S}^{(n)}$. The normalized distribution is

$$
q_{i, j}^{(n)}(S)=D(\alpha)=\frac{1}{\sigma_{S}\left(2+\sigma_{S}^{2}\right) \cos ^{4} \alpha} \exp \left[-\left(\frac{\tan (\alpha)}{\sigma_{S}}\right)^{2}\right]
$$

where $\sigma_{S}$ is a parameter which controls the angular spread of the specular spike. This distribution can be used to model the shape of both the specular spike and the specular lobe. Our model of the Lambertian reflectance process assumes that the observed intensity values follow a Gaussian distribution with variance $\sigma_{L}^{2}$. The mean intensity is $\boldsymbol{M}^{(n)} \cdot \boldsymbol{L}$. Under these assumptions we can write

$$
q_{i, j}^{(n)}(L)=\frac{1}{\sqrt{2 \pi} \sigma_{L}} \exp \left[-\frac{1}{2}\left(\frac{\left.E(i, j)-\boldsymbol{M}^{(n)}(i, j) \cdot \boldsymbol{L}\right)}{\sigma_{L}}\right)^{2}\right]
$$

Our model for the surface normal smoothness prior is based on the average value of the inner product of the surface normal at the location $(i, j)$ with the surrounding field of PM surface normals. We write

$$
P_{i, j}^{(n)}(\Lambda)=\frac{1}{2\left|G_{i, j}\right|}\left[\left|G_{i, j}\right|+\sum_{(k, l) \in G_{i, j}} \boldsymbol{N}_{\Lambda}^{(n)}(i, j) \cdot \boldsymbol{M}^{(n)}(k, l)\right]
$$

When the PM surface normals from the neighbourhood $G_{i, j}$ are aligned in the direction of $\boldsymbol{N}_{\Lambda}^{(n)}(i, j)$, then $P_{i, j}^{(n)}(\Lambda)=1$, the larger the misalignment then the smaller the value of smoothness prior.

\section{Specular SFS Algorithm}

Having described the Bayes framework and the associated two-mode reflectance model, we are now in a position to develop a practical SFS algorithm.

We commence by initialising the algorithm. The initial Lambertian surface normal $\boldsymbol{N}_{L}^{(0)}$ is constrained to lay on the irradiance cone in the direction of the image gradient. The subsequent iterative steps of the algorithm are as follows: 
- 1: The field of PM surface normals $\boldsymbol{M}^{(n)}$ (initially equal to $\boldsymbol{N}_{L}^{(0)}$ ) is subjected to local smoothing. Here we use the curvature sensitive smoothing method [16]. The smoothed PM surface normal is denoted by $\boldsymbol{M}_{R}^{(n)}(i, j)$.

- 2: We update the current estimate of the Lambertian surface normal by projecting the smoothed PM surface normal onto the nearest location on the irradiance cone. This gives us the revised surface normal of $\boldsymbol{N}_{L}^{(n)}$.

- 3: With $\boldsymbol{M}_{R}^{(n)}(i, j)$ to hand we compute the conditional measurement densities $q_{i, j}^{(N)}(L)$ and $q_{i, j}^{(N)}(S)$ for the two reflectance modes. Taking $\boldsymbol{M}_{R}^{(n)}(i, j)$, $\boldsymbol{N}_{L}^{(n)}$ and $\boldsymbol{N}_{S}^{(n)}$, we compute the smoothness priors $P_{i, j}^{(n)}(L)$ and $P_{i, j}^{(n)}(S)$, and also the updated a posteriori probabilities for both reflectance modes.

- 4: Using $\boldsymbol{N}_{L}^{(n)}$ and $\boldsymbol{N}_{S}^{(n)}$ and the updated a posteriori probabilities, we compute the new PM surface normal $\boldsymbol{M}^{(n+1)}(i, j)$ and we return to step 1.

The steps of the algorithm are summarised in Figure 1b. The PM surface normals delivered by our SFS algorithm can be used for the purposes of reconstructing the specular intensity component. The reason for doing this is that the specular intensity may be removed from the original image intensity to give a corrected Lambertian image. We use the T-S model [14] to reconstruct the specular intensity component $I_{S}$. With the reconstructed specular intensity to hand, we can compute the matte reflectance component $I_{L}(i, j)=E(i, j)-I_{S}(i, j)$. By re-applying the SFS algorithm to this corrected intensity image, we aim to recover improved surface normal estimates, free of the high curvature artifacts of specular highlights.

\section{Correcting for Limb-Brightening}

As mentioned earlier, there may also surface brightness anomalies due to rough reflectance from the limbs of objects. Our aim in this section is to show how the Oren and Nayar model [11] can be used to further correct the images obtained by specular subtraction for limb-brightening. According to this model, for a point on a rough surface with roughness parameter of $\sigma$ and illuminant incidence direction $\left(\theta_{i}, \phi_{i}\right)$ and reflectance direction $\left(\theta_{r}, \phi_{r}\right)$, the reflectance functions is

$$
L_{r}\left(\theta_{i}, \theta_{r}, \phi_{r}-\phi_{i} ; \sigma\right)=\frac{\rho}{\pi} E_{0} \cos \left(\theta_{i}\right)\left(A+B \max \left[0, \cos \left(\phi_{r}-\phi_{i}\right)\right] \sin (\alpha) \tan (\beta)\right)
$$

where $A=1.0-0.5 \frac{\sigma^{2}}{\sigma^{2}+0.33}, B=0.45 \frac{\sigma^{2}}{\sigma^{2}+0.09}$ and $\alpha=\max \left[\theta_{i}, \theta_{r}\right], \beta=$ $\min \left[\theta_{i}, \theta_{r}\right]$. It is important to note that the model reduces to the Lambertian case when $\sigma=0$. Here, we aim to utilize this model to deduce a corrected Lambertian reflectance image from the matte component delivered by our specular subtraction method. To do this, we assume that $\sigma$ is almost constant and the reflectance measurements are obtained in the plane of incidence $\left(\phi_{r}=\phi_{i}=0\right)$. We also confine our attention to the case where the angle between the light source and the viewing directions is small, i.e. $\theta_{r}=\theta_{i}=\theta$. With these two restrictions, we can write $\cos \left(\phi_{r}-\phi_{i}\right)=1$ and $\alpha=\beta=\theta$. Hence, the non-specular (or matte) intensity predicted by the simplified Oren-Nayar (O-N) model is 


$$
I_{M}(i, j)=A \cos \theta+B \sin ^{2} \theta
$$

Hence, the matte intensity consists of two components. The first of these is a Lambertian component $A \cos \theta$. The second is the non-Lambertian component $B \sin ^{2} \theta$ which takes on its maximum value where $\theta=\frac{\pi}{2}$, i.e. close to the occluding boundary. To perform Lambertian correction, we proceed as follows. At every pixel location, we use Equation (5) to estimate the angle $\theta$ using the subtracted matte intensity and solving the resulting quadratic equation in $\cos \theta$.

The solution is

$$
\cos \theta=\frac{A \mp \sqrt{A^{2}-4 B\left(I_{M}(i, j)-B\right)}}{2 B}
$$

We take the sign above which results in a value of $A \cos \theta$ which is closest to the matte intensity $I_{M}$ (in the majority of cases this involves taking the solution associated with the minus sign). This hence allows us to reconstruct the corrected Lambertian reflectance image $I_{L}=A \cos \theta$. It also gives us an estimate of the opening angle of the Lambertian reflectance cone. This can then be used in the Worthington and Hancock SFS scheme which assumes the Lambertian reflectance model to recover improved surface normal estimates.

In Figure $2 \mathrm{a}$ we show the Lambertian reflectance $\cos \theta$ (equation 6) as a function of the roughness parameter $\sigma$ and the matte intensity $I_{M}$. When the roughness is zero, then the Lambertian and matte intensities are equal to one another. When the roughness increases, then the departures from Lambertian reflectance become more marked. In Figure $2 \mathrm{~b}$ we plot the ratio $\frac{I_{L}}{I_{M}}$ as a function of the incidence angle $\theta$. The different curves are for different values of the roughness parameter $\sigma$. For zero roughness, the ratio is flat, i.e. the reflectance is purely Lambertian. As the roughness increases, then so the value of the ratio decreases with increasing incidence angle. For normal incidence, the ratio is always unity, i.e. the reflectance is indistinguishable from the Lambertian case, whatever the value of the roughness parameter is.

\section{Experiments}

The images used in our experiments have been captured using an Olympus 10E camera. The objects studied are made of white porcelain and are hence shiny. Each object has been imaged under controlled lighting conditions in a darkroom. The objects have been illuminated using a single collimated tungsten light source. The light source direction is recorded at the time the images are captured. To ground-truth the surface highlight removal process, we have used a pair of polaroid filters. We have placed the first filter between the light source and the illuminated object. The second filter was placed between the illuminated object and the camera. For each object we have collected a pair of images. The first of these is captured when the second filter (i.e. the one between the camera and the object) is rotated until there is maximal extinction of the observed specularities. The second image is obtained when the polaroid is rotated through $90^{\circ}$, i.e. there is minimal extinction of the specularities. We refer to the polarisation conditions of the former image as "uncrossed" and of the latter as "crossed". 
In Figure 3 we show the results obtained for three of the objects used in our study. The objects are a porcelain bear, a porcelain vase and a porcelain urn. The top row of the Figure shows the images obtained with uncrossed polaroids while the second row shows the images obtained with crossed polaroids. The third row shows the difference between the crossed and uncrossed polaroid images. The strongest differences occur at two different locations. Firstly, there are the positions of specularities. From the uncrossed polaroid images it is clear that there are several quite small specular reflections across the surface of the bear. The vase has larger specularities on the neck and the centre of the bulb. The urn has a complex pattern of specularities around the handles. From the crossed polaroid images it is clear that most of the specular structure is removed. The second feature in the difference images are the locations of occluding object limbs, where oblique scattering occurs.

In the fifth row of Figure 3, we show the reconstructed specular intensity obtained using the T-S model, i.e. $I_{S}$, The fourth row shows the matte images $I_{M}$ obtained after specularity subtraction. Turning our attention to the matte images and the specular images, it is clear that for each of the objects the specular structure is cleanly removed and the matte appearance is close to that obtained with the crossed polaroids. Also, the pattern of specularities obtained in each case corresponds to that obtained by subtracting the crossed polaroid images from the uncrossed polaroid images.

In Figure 4 we investigate the shape information recoverable. The top row shows the Lambertian images after correction for rough limb reflectance using the simplified O-N model, i.e. $I_{L}$. In the second row of the Figure we show the needle maps obtained when the SFS is applied to the corrected Lambertian images $\left(I_{L}\right)$ appearing in the top row of this Figure. The third row of Figure 4 shows the difference in needle-map directions for the matte $\left(I_{M}\right)$ and Lambertian images $\left(I_{L}\right)$. Here the main differences occur at the limbs of the objects. The fourth row of Figure 4 show the curvedness estimated using the surface normals delivered by the corrected Lambertian images. In the case of the urn the ribbed structure emerges well. The complex surface structure of the bear, in particular the boundaries of the arms and legs, is clearly visible. For the urn the symmetric structure of the neck and the bulb is nicely preserved.

In Figure 5 we provide some analysis of the different reflectance models used in our experiments. In the left hand panel of the Figure, the solid curve is the intensity cross-section along a horizontal line crossing the uncrossed image of the neck of the vase shown in Figure 3. The dashed curve shows the matte image $I_{M}$ while the dotted curve is the specular component $I_{S}$. The specularity on the neck is clearly visible as a peak in the solid curve. This peak is cleanly subtracted in the matte (dashed) curve. In the right-hand panel we focus on the corrected Lambertian image. Here the solid curve is the matte reflectance $I_{M}$. The dashed curve is the corrected Lambertian reflectance $I_{L}$. The differences between the two curves are small except at the limbs of the object. To examine the effect of the model in more detail, the dotted curve shows the ratio of corrected Lambertian and matte reflectance $\rho=\frac{I_{L}}{I_{M}}$. The ratio drops rapidly towards zero as the limbs 
are approached. Also shown on the plot as a dash-dot curve is the predicted value of the ratio based on the assumption that the object has a circular cross-section. If $x$ is the distance from the centre and $r$ is the radius of the circle, then value of the ratio at a distance $x$ from the centre is $\rho(x)=\frac{A \sqrt{1-\left(\frac{x}{r}\right)^{2}}}{A \sqrt{1-\left(\frac{x}{r}\right)^{2}}+B\left(\frac{x}{r}\right)^{2}}$. This simple model is in reasonable agreement with the empirical data.

\section{Conclusions}

In this paper we have shown how to use shape-from-shading to perform photometric correction of images of shiny objects. Our approach is to use estimated surface normal directions together with reflectance models for specular and rough reflectance to perform specularity removal and rough limb-correction. Specularities are modelled using the T-S model while the rough limb brightening is modelled using the $\mathrm{O}-\mathrm{N}$ model. We commence by using an iterated conditional modes algorithm to extract surface normals using a mixture of specular and matte reflectance directions. The resulting surface normals are used to perform specularity subtraction. Finally, we correct the residual matte reflectance component for rough limb scattering using the O-N model. The resulting corrected Lambertian images can be used as input to a conventional shape-from-shading algorithm and result in improved recovery of object-geometry.

\section{References}

1. P. Beckmann and A. Spizzochino, The Scattering of Electromagnetic Waves from Rough Surfaces, Pergamon, New York, 1963. 576

2. A. Blake and H. Bulthoff, "Shape from Specularities: computation and psychophysics," Phil Trans R. Soc. Lond. B, Vol. 331, pp. 237-252, 1991.

3. K. Dana, S. Nayar, B. Van Ginneken, J. Koenderink, "Reflectance and Texture of Real-World Surfaces," CVPR, pp. 151-157, 1997. 577

4. G. Healey and T. Binford "Local shape from specularity" ICCV, pp. 151-160, 1987. 576

5. B. K. P. Horn and M. J. Brooks, "The Variational Approach to Shape from Shading," CVGIP, Vol. 33, No. 2, pp. 174-208, 1986. 576

6. C. C. J. Kuo and K. M. Lee, "Shape from Shading With a Generalized Reflectance Map Model," CVIU, Vol. 67, No. 2, pp. 143-160, 1997. 576

7. S. Lin and S. W. Lee, "Estimation of Diffuse and Specular Appearance," ICCV, pp. 855-860, 1999. 577

8. S. Magda, D. Kriegman, T. Zickler and P. Belhumeur, "Beyond Lambert: Reconstructing Surfaces with Arbitrary BRDFs," ICCV, Vol. 2, pp. 391-399, 2001. 577

9. S. K. Nayar, K. Ikeuchi and T. Kanade, "Surface Reflection: Physical and Geometrical Perspectives," PAMI, Vol. 13, No. 7, pp. 611-634, 1991. 576

10. S. K. Nayar, X. Fang and T. Boult, "Removal of specularities using color and polarization," CVPR, pp. 583-590, 1993. 577

11. M. Oren and S. K. Nayar, "Generalization of the Lambertian Model and Implications for Machine Vision," IJCV, vol. 14, No. 3, pp. 227-251, 1995. 577, 580 
12. H. Ragheb, and E. R. Hancock, "Separating Lambertian and Specular Reflectance Components using Iterated Conditional Modes," BMVC, pp. 541-552, 2001. 578

13. H. D. Tagare and R. J. P. deFigueiredo, "A Theory of Photometric Stereo for a Class of Diffuse Non-Lambertian Surfaces," PAMI, Vol. 13, No. 2, pp. 133-151, 1991. 576

14. K. Torrance and E. Sparrow, "Theory for Off-Specular Reflection from Roughened Surfaces," JOSA, Vol. 57, pp. 1105-1114, 1967. 576, 577, 580

15. L. B. Wolff, "On The Relative Brightness of Specular and Diffuse Reflection," CVPR, pp. 369-376, 1994. 577

16. P. L. Worthington and E. R. Hancock, "New Constraints on Data-closeness and Needle-map consistency for SFS," PAMI, Vol. 21, No. 11, pp. 1250-1267, 1999. 580
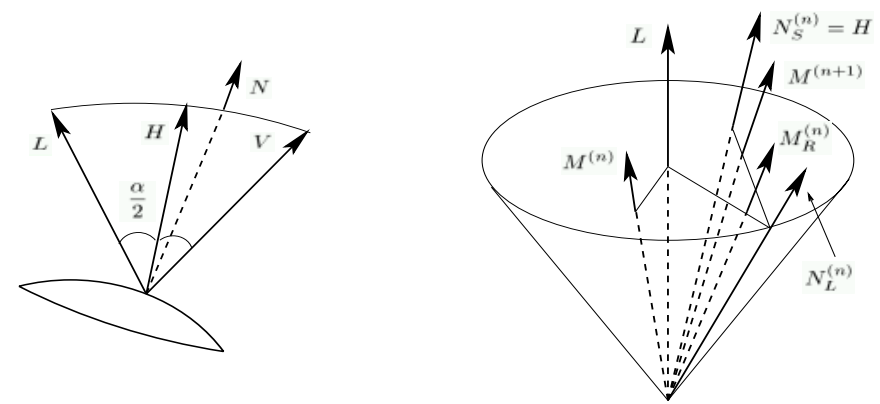

Fig. 1. Geometry of the specular reflectance and the surface normal update process
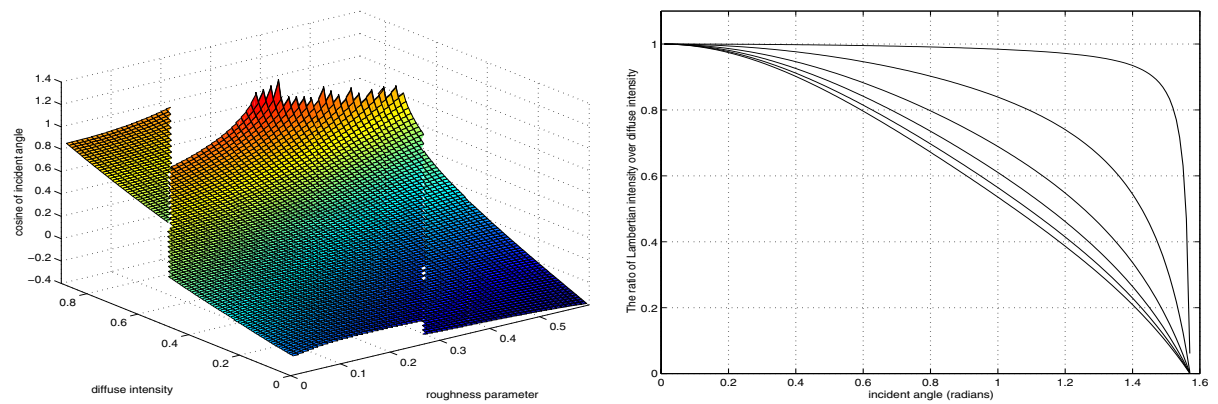

Fig. 2. Plots showing the behaviour of the Oren and Nayar model for rough surfaces 

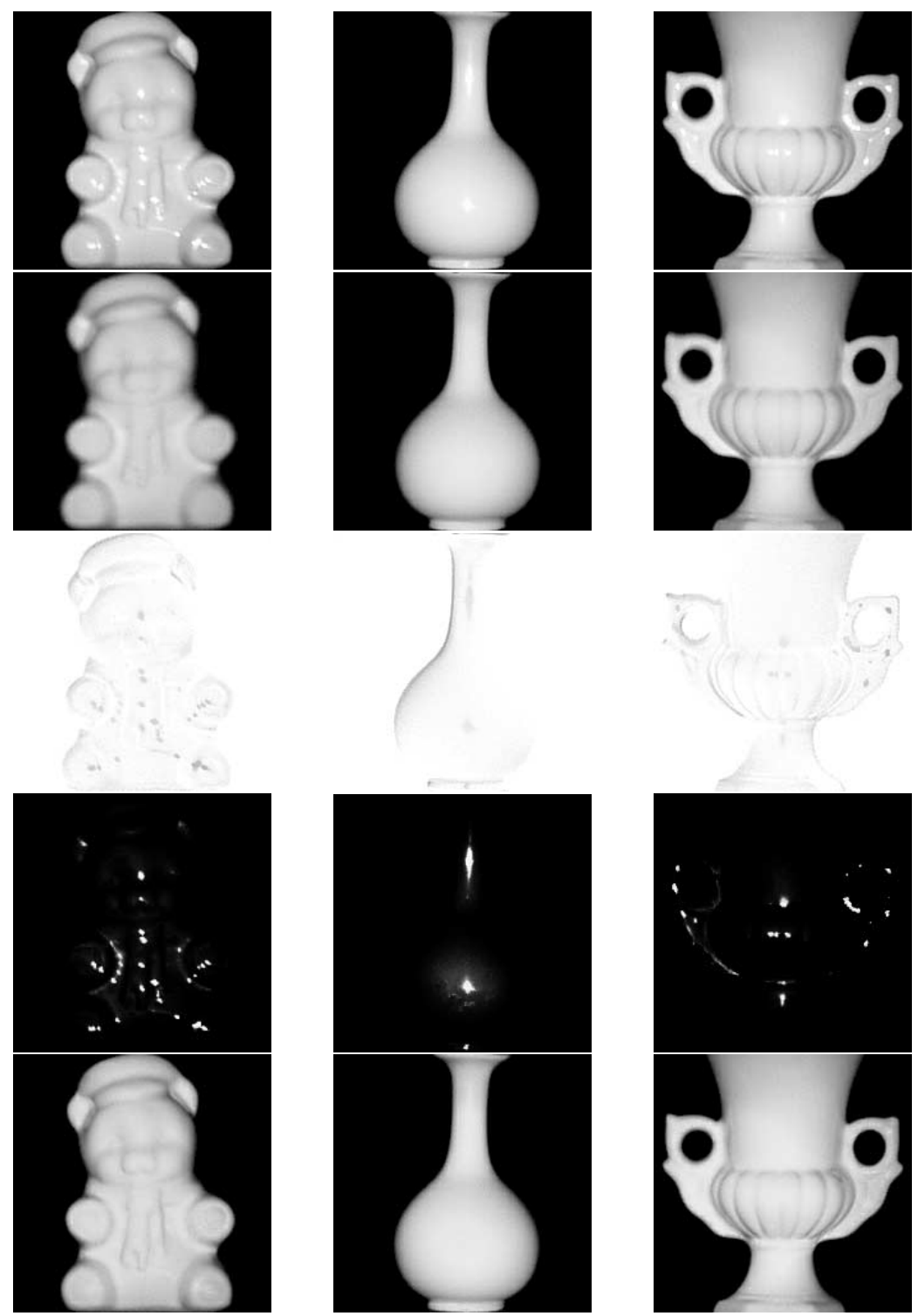

Fig. 3. Applying our specular SFS to separate the specular and matte components 

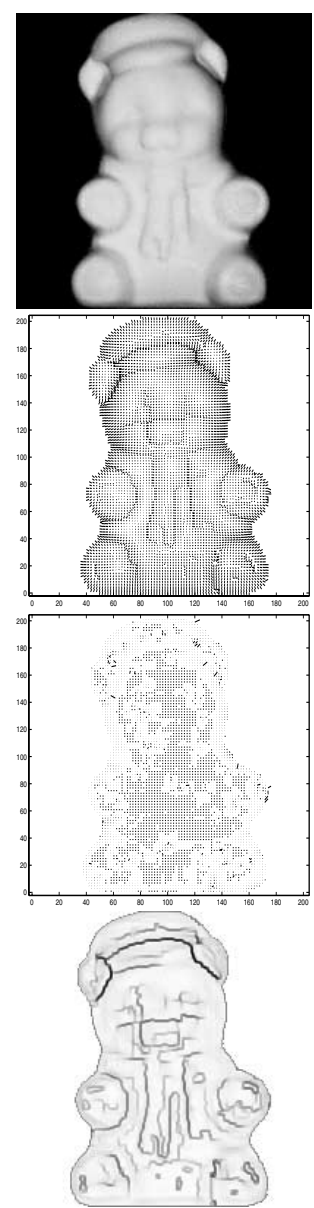
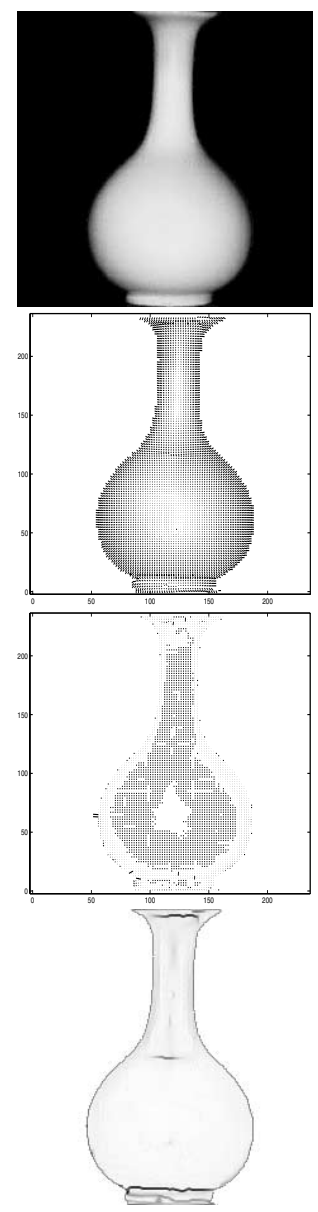
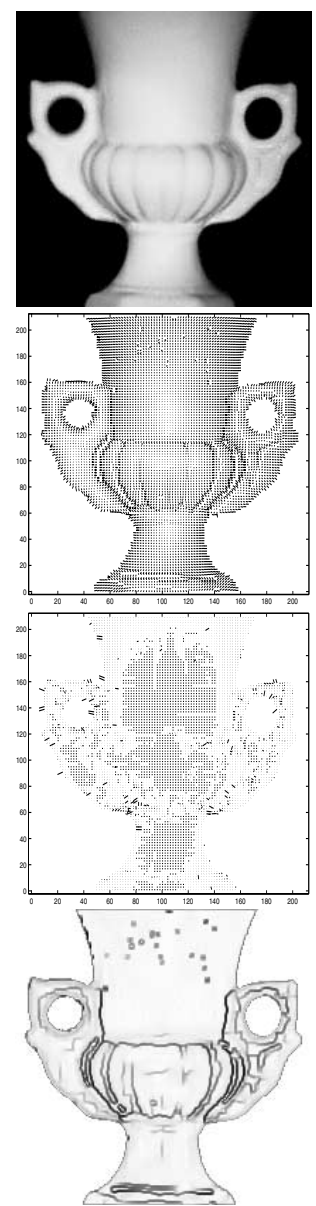

Fig. 4. Surface normals obtained by running SFS over matte and Lambertian images
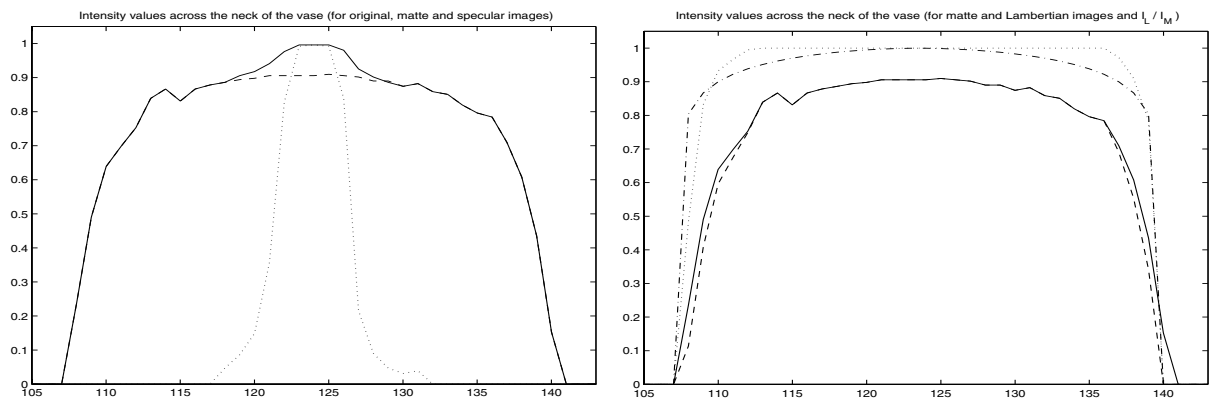

Fig. 5. Intensity plots for different reflectance components across the neck of the vase 